\title{
Effect of B-vitamins and n-3 PUFA supplementation for 5 years on blood pressure in patients with CVD
}

\author{
Fabien Szabo de Edelenyi ${ }^{1}$, Anne-Claire Vergnaud ${ }^{1}$, Namanjeet Ahluwalia ${ }^{1}$, Chantal Julia ${ }^{1}$, \\ Serge Hercberg ${ }^{1,2}$, Jacques Blacher ${ }^{1,3}$ and Pilar Galan ${ }^{1 *}$ \\ ${ }^{1}$ UMR U557 INSERM, U1125 INRA, CNAM, Université Paris 13, CRNH IdF, SMBH, 74, rue Marcel Cachin, F-93017 Bobigny, \\ France \\ ${ }^{2}$ Département de Santé publique, Hôpital Avicenne, F-93017 Bobigny, France \\ ${ }^{3}$ Université Paris-Descartes, Faculté de Médecine, AP-HP, Hôtel-Dieu, Centre de Diagnostic et Thérapeutique, Paris, France
}

(Received 21 January 2011 - Revised 7 June 2011 - Accepted 7 June 2011 - First published online 1 August 2011)

\section{Abstract}

Certain epidemiological and experimental studies suggest that $n-3$ fatty acids and folate can reduce blood pressure (BP). We investigated the effect of a daily supplementation with dietary doses of B-vitamins or $n-3$ fatty acids for 5 years on BP in patients with a history of CVD who participated in the Supplémentation en Folates et Omega-3 trial. The patients ( $n$ 2501; 1987 men and 514 women) were randomly assigned in a $2 \times 2$ factorial design to one of four groups: B-vitamins (5-methyl-THF $(560 \mu \mathrm{g})$; vitamin $\mathrm{B}_{6}$ ( $\left.3 \mathrm{mg}\right)$ and vitamin $\mathrm{B}_{12}$ $(20 \mu \mathrm{g})$ ) and a placebo capsule for $n-3$ fatty acids; $n-3$ fatty acids (600 mg of EPA and DHA at a ratio of $2: 1)$ and a placebo capsule for B-vitamins; both B-vitamins and $n-3$ fatty acids; or placebo capsules for both treatments. The patients took two capsules daily in a double-blind manner for a median duration of 4.7 years. At baseline and annual examination for 5 years, the patients underwent a clinical examination where BP and clinical and biological parameters were assessed. No effect of supplementation with either $n$ - 3 PUFA or B-vitamins on BP was observed in crude and adjusted multivariate models. Change in BP was not associated with change in homocysteine. In conclusion, the present results do not support the routine use of dietary supplements containing B-vitamins, or of $n-3$ fatty acids, to reduce BP in people with prior CVD.

Key words: Hypertension: $n$-3 Fatty acids: Folate: Randomised controlled trials: Blood pressure

Hypertension has been shown to be an independent, quantitative and reversible risk factor for CVD, including stroke, $\mathrm{CHD}$, peripheral artery disease and cardiac failure, in primary and secondary prevention studies ${ }^{(1)}$. Relatively small blood pressure (BP) reductions of about $5 \mathrm{mmHg}$ in systolic BP can reduce the risk of strokes by one-third and coronary events by one-fifth ${ }^{(2)}$. The potential of both $n-3$ PUFA and B-vitamins in reducing $\mathrm{BP}$ was hypothesised several years ago ${ }^{(3,4)}$; yet, no clear and definitive answer has been found in primary and secondary prevention trials ${ }^{(5-9)}$.

A lowering effect of $n$ - 3 fatty acids on BP has been described in the literature, but the results are quite heterogeneous ${ }^{(5,6)}$. In addition, most studies used high doses of $n-3$ fatty acids ${ }^{(10)}$. $n-3$ Fatty acids can influence BP through various mechanisms including an interaction with angiotensin II. Indeed, $n-3$ PUFA reduce angiotensin-converting enzyme activity as well as angiotensin II formation, resulting in an improved vasodilation and arterial compliance of both small and large arteries ${ }^{(11)}$. Other mechanisms of the action proposed are reduced production of the vasoconstrictor thromboxane A2, increased synthesis of NO and an effect on autonomic nerve function ${ }^{(12)}$

With regard to B-vitamins, and particularly folic acid, a lowering effect on BP has been described extensively in the literature $e^{(7,9,13-16)}$. The effect of folic acid on BP could be due to its action on homocysteine. Plasma homocysteine levels have been positively associated with BP in crosssectional studies ${ }^{(17,18)}$. Furthermore, in several intervention studies, supplementation with folic acid or 5-methyltetrahydrofolate decreased homocysteine concentration ${ }^{(19)}$

Although the role of B-vitamins and $n-3$ fatty acids on $\mathrm{BP}$ is mechanistically grounded, this has not been consistently shown in intervention studies, which have usually been of short-to-medium duration and have generally employed supplements at high doses.

The Supplementation in Folate (and B-vitamins) or $n-3$ (Supplémentation en Folates et Omega-3; SU.FOL.OM3) trial $^{(20)}$ provides a unique opportunity to assess in a large sample of patients with a history of CVD, the impact of

Abbreviations: BP, blood pressure; SU.FOL.OM3, Supplémentation en Folates et Omega-3.

*Corresponding author: Dr P. Galan, fax +33 1483889 31, email p.galan@uren.smbh.univ-paris13.fr 
supplementation with B-vitamins (5-methyl-tetrahydrofolate, vitamin $\mathrm{B}_{12}$ and vitamin $\mathrm{B}_{6}$ ) and/or $n$-3 PUFA for 5 years on BP. The primary objective of the SU.FOL.OM3 trial was to examine the effect of these interventions on the recurrence of CVD outcomes. We conducted post hoc analyses in the SU.FOL.OM3 cohort to study the effect of supplementation with B-vitamins and/or $n$-3 fatty acids for a long duration ( 5 years) on BP in a population with CVD.

\section{Subjects and methods}

\section{Study design}

The SU.FOL.OM3 trial is a multi-centre, double-blind randomised trial with a placebo-controlled factorial design that has evaluated the separate and combined effects of daily supplementation with B-vitamins and $n-3$ PUFA on the prevention of $\mathrm{CVD}^{(20)}$. The detailed protocol has been described previously ${ }^{(20)}$. Briefly, patients with CVD (myocardial infarction, stroke and unstable angina) were randomly assigned in a $2 \times 2$ factorial design to one of four groups: B-vitamins (5-methyl-THF $(560 \mu \mathrm{g})$; vitamin $\mathrm{B}_{6}(3 \mathrm{mg})$ and vitamin $\mathrm{B}_{12}$ $(20 \mu \mathrm{g}))$ and a placebo capsule for fatty acids; $n-3$ fatty acids (600 mg of EPA and DHA at a ratio of 2:1 in the form of TAG) and a placebo capsule for B-vitamins; both B-vitamins and $n$ - 3 fatty acids; or placebo capsules for both treatments. The patients took two capsules daily in a double-blind manner for 5 years.

B-vitamins placebo and $n$ - 3 fatty acids placebo capsules were developed to maintain the blindness of the trial, and were manufactured to be indistinguishable from their corresponding treatment capsule by external colour, size or taste (use of fish oil flavour for $n$ - 3 placebo). The supplements were provided by Merck Eprova AG (Schaffhausen, Switzerland) (for 5-methyl-THF), Roche Laboratories (Basel, Switzerland) (for vitamin $\mathrm{B}_{6}$ and vitamin $\mathrm{B}_{12}$ ) and Pierre Fabre (Ramonville, France) (for $n-3$ PUFA). The gelatine placebo capsules contained liquid paraffin, and for the $n-3$ placebo the capsules additionally contained fish oil flavour. The gelatine capsules were manufactured by Catalent Pharma Solutions (Beinheim, France; Swindon, UK).

Randomisation was performed in blocks using a computerbased randomisation programme and stratified by three age categories (44-54, 55-64 and 65-80 years), sex, inclusion criteria for CVD and recruitment centre. The present study was conducted according to the guidelines laid down in the Declaration of Helsinki, and all the procedures involving human subjects were approved by the ethics committee 'Comité Consultatif pour la Protection des Personnes se prêtant à la Recherche Biomédicale' (CCPPRB no. 1933) of Paris-Cochin, and the data protection board 'Comité National Informatique et Liberté' (CNIL no. 901230). Written informed consent was obtained from all patients. All the analyses and interpretation were carried out independent of the organisations that funded the study. The present trial was registered under the no. ISRCTN41926726 in Current Controlled Trials (http://www.controlled-trials.com/ ISRCTN41926726).

\section{Study population}

Participants with a history of CVD were recruited via a network of 417 cardiologists, neurologists or other physicians throughout France. Men and women aged $45-80$ years who had a history of an acute coronary or cerebral ischaemic event occurring within 1-12 months before randomisation were eligible to participate.

Post hoc analyses were performed for all the patients who were included in the primary analyses for the SU.FOL.OM3 study $^{(20)}$. Among a total of 2501 participants (1987 men and 514 women), the $2 \times 2$ random assignment yielded four groups: B-vitamins and placebo for $n$-3 fatty acids ( $n$ 622); $n$-3 fatty acids and placebo for B-vitamins ( $n$ 633); both active treatments ( $n$ 620); and placebo for both treatments ( $n$ 626). Among the 2501 participants, 2170, 2066, 1924, 1618 and 1184 individuals attended the non-mandatory annual examinations at years $1-5$, respectively. The median follow-up time was $4 \cdot 7$ years.

\section{Measurements}

$\mathrm{BP}$ was measured at baseline and during annual examinations for the subsequent 5 years. BP was recorded by trained study staff for each patient in the sitting position, after a 5 min rest, with a semi-automatic device (Digital blood pressure monitor OMROM UA-787; OMRON Corporation, Kyoto, Japan) using standardised protocols. We took two measurements. In the case of a BP difference greater than $10 \mathrm{mmHg}$ for either systolic BP or diastolic BP, BP was measured again after a $5 \mathrm{~min}$ rest. The last two $\mathrm{BP}$ values were averaged.

BMI $\left(\mathrm{kg} / \mathrm{m}^{2}\right)$ was calculated at each annual examination from the corresponding measurements of height and weight recorded by the trained staff following standard protocols. Data on age, level of education (elementary school, secondary school and university or equivalent), smoking status (current smokers, former smokers and non-smokers) and medication use were collected at inclusion via questionnaires.

Blood samples were obtained at baseline and at each annual follow-up examination after a $12 \mathrm{~h}$ fast. All biochemical measurements were centralised. Total cholesterol, TAG, and HDL- and LDL-cholesterol were measured by the enzymatic method with the use of the C8000 System (Abbott, Rungis, France); plasma homocysteine, serum folate and vitamin $\mathrm{B}_{12}$ were measured by a competitive immunoassay with direct chemiluminescence detection; and pyridoxal 5'-phosphate (circulating form of vitamin $\mathrm{B}_{6}$ ) was determined in the plasma by HPLC.

Plasma LDL-cholesterol $>2.55 \mathrm{mmol} / \mathrm{l}$, plasma homocysteine $>15 \mu \mathrm{mol} / 1$ and $\mathrm{BMI}>25 \mathrm{~kg} / \mathrm{m}^{2}$ were used to define hypercholesterolaemia, hyperhomocysteinaemia and overweight, respectively. Independently of anti-hypertensive drug prescription, hypertension was considered to be present whenever the achieved systolic and the diastolic BP were higher than 140 and $90 \mathrm{mmHg}$, respectively.

Compliance was assessed by means of self-reported questionnaires at 6-month intervals (or by a telephone interview by study physicians). The patients were considered compliant 
if they took at least $80 \%$ of their allocated treatment. In addition, change in plasma B-vitamins ( $n$ 2147) was also examined. Fatty acid composition of plasma lipids was determined on a subgroup ( $n$ 682) as well, at baseline and 1 year post-intervention, by GC of the corresponding methyl esters.

\section{Statistical analysis}

To examine the effect of the intervention, mixed linear models with repeated measures were built using the difference in $\mathrm{BP}$ between two successive examinations as the dependent variable. This model was repeated with adjustment for subject characteristics at baseline (inclusion criteria for CVD, sex, level of education and age), and clinical (BP, number of anti-hypertensive drugs and BMI) and biological (plasma homocysteine and cholesterol concentrations) data assessed at the beginning of each period, as well as change in clinical and biological variables between two successive measurements. All two-by-two interactions between independent variables were tested in these mixed models. Bonferroni's correction for multiple testing was applied. None of the interactions were significant.

To illustrate the effect of supplements (B-vitamins or $n-3$ fatty acids) on the evaluation of $\mathrm{BP}$, we first obtained combined $n-3$ or combined B-vitamins groups ${ }^{(20)}$ by merging the groups receiving the $n-3$ supplement (either alone or with B-vitamins) and the groups receiving B-vitamins (either alone or with $n$-3 fatty acids), respectively. The mixed linear model with repeated measures was run with the combined $n-3$ group and the combined B-vitamins groups and interaction terms ( $n$ - $3 \times$ time, B-vitamins $\times$ time, $n-3 \times \mathrm{B}$-vitamins and $n-3 \times$ B-vitamins $\times$ time). BP at successive measurements was defined as the dependent variable. Interaction of intervention group $\times$ time with the inclusion criteria for CVD, sex, age, overweight status, hyperhomocysteinaemia and BP at baseline was tested and found non-significant.

All statistical analyses were performed using SAS (SAS Institute, Inc., Cary, NC, USA) version 9.1 software.

\section{Results}

Patient characteristics at baseline are shown for all four supplementation groups in Table 1 . The participants were 61.3 (SD 9.0) years old and most were male (79.5\%). Among the three inclusion criteria, myocardial infarction was the most common reason for inclusion. At inclusion, 979 participants had a high achieved BP (39.2\%); average systolic $\mathrm{BP}$ was 133.4 (SD 21.4) and diastolic BP was 83.2 (SD 12.3.) Of the participants, $74 \%$ were overweight; average BMI was 27.6 (SD 4.0); 53\% had hypercholesterolaemia and $29 \cdot 3 \%$ had hyperhomocysteinaemia. The participants with a high achieved BP at baseline were older, had higher BMI and had higher plasma total cholesterol and homocysteine concentrations than individuals with controlled $\mathrm{BP}$ $(P<0 \cdot 0001)$

Compliance with the supplementation regimen was high. The overall response rate for return of completed questionnaires was 99, 96,94 and 95\% at 6,12 and 24 months and at the end of the trial, respectively; $86 \%$ of the respondents were considered compliant with the study medication (took $>80 \%$ of assigned capsules) and compliance was similar (approximately 86\%) in all four treatment groups.

As reported previously ${ }^{(20)}$, the median (interquartile range) baseline concentration for plasma folate was $6.7 \quad(5 \cdot 2-$ 8.7) $\mathrm{ng} / \mathrm{ml}$, vitamin $\mathrm{B}_{12}$ was $365 \quad(296-463) \mathrm{pg} / \mathrm{ml}$ and homocysteine was $12.8(10 \cdot 8-15 \cdot 8) \mu \mathrm{mol} / \mathrm{l}$; EPA represented $1 \cdot 16(0 \cdot 81-1 \cdot 73) \%$ of total fatty acids and DHA represented $2 \cdot 58(2 \cdot 05-3 \cdot 28) \%$ of total fatty acids at baseline examination. Baseline plasma concentrations for B-vitamins, homocysteine and $n$ - 3 fatty acids were not statistically different among the four groups. Allocation to B-vitamins was associated with a $20 \%(2.5 \mu \mathrm{mol} / \mathrm{l})$ reduction in median plasma homocysteine concentration after 1 year and $19 \%(2 \cdot 7 \mu \mathrm{mol} / \mathrm{l})$ reduction at the end of the trial compared with placebo. Allocation to B-vitamins was associated with a $146 \%$ increase in serum folate, $35 \%$ increase in serum vitamin $\mathrm{B}_{12}$ and $116 \%$ increase in serum vitamin $\mathrm{B}_{6}$. Supplementation with $n$ - 3 fatty acids was

Table 1. Baseline characteristics of participants

Mean values, standard deviations, number of participants and percentages

\begin{tabular}{|c|c|c|c|c|c|c|c|c|c|c|c|c|}
\hline & \multicolumn{3}{|c|}{ B-vitamins } & \multicolumn{3}{|c|}{$n-3$ PUFA } & \multicolumn{3}{|c|}{$\begin{array}{c}\text { B-vitamins }+n-3 \\
\text { PUFA }\end{array}$} & \multicolumn{3}{|c|}{ Placebo } \\
\hline & Mean & SD & $\%$ & Mean & SD & $\%$ & Mean & SD & $\%$ & Mean & SD & $\%$ \\
\hline$n$ & \multicolumn{3}{|c|}{622} & \multicolumn{3}{|c|}{633} & \multicolumn{3}{|c|}{620} & \multicolumn{3}{|c|}{626} \\
\hline Age (years) & 61.4 & $8 \cdot 7$ & & $61 \cdot 3$ & $9 \cdot 2$ & & $61 \cdot 3$ & $9 \cdot 3$ & & 61.4 & 8.9 & \\
\hline Men & & & $79 \cdot 9$ & & & $79 \cdot 2$ & & & $79 \cdot 5$ & & & $79 \cdot 2$ \\
\hline \multicolumn{13}{|l|}{ Inclusion criteria for CVD } \\
\hline Myocardial infarction & & & $46 \cdot 3$ & & & $47 \cdot 4$ & & & $45 \cdot 2$ & & & $45 \cdot 0$ \\
\hline Unstable angina & & & $27 \cdot 0$ & & & $29 \cdot 2$ & & & $28 \cdot 4$ & & & $29 \cdot 4$ \\
\hline Stroke & & & $26 \cdot 7$ & & & 23.4 & & & $26 \cdot 4$ & & & $25 \cdot 6$ \\
\hline \multicolumn{13}{|l|}{ Smoking } \\
\hline Current smokers & & & $10 \cdot 9$ & & & $11 \cdot 3$ & & & $11 \cdot 4$ & & & $10 \cdot 1$ \\
\hline Former smokers & & & 61.4 & & & $59 \cdot 2$ & & & $61 \cdot 5$ & & & 63.9 \\
\hline Non-smokers & & & $27 \cdot 7$ & & & 29.5 & & & $27 \cdot 1$ & & & $26 \cdot 0$ \\
\hline \multicolumn{13}{|l|}{ Blood pressure $(\mathrm{mm} \mathrm{Hg})$} \\
\hline Systolic & $133 \cdot 5$ & $22 \cdot 2$ & & $134 \cdot 2$ & $22 \cdot 0$ & & $133 \cdot 1$ & $21 \cdot 3$ & & $132 \cdot 7$ & $20 \cdot 0$ & \\
\hline Diastolic & 83.4 & $12 \cdot 7$ & & $83 \cdot 8$ & $12 \cdot 3$ & & $83 \cdot 1$ & $12 \cdot 2$ & & 82.4 & 11.9 & \\
\hline $\mathrm{BMI}\left(\mathrm{kg} / \mathrm{m}^{2}\right)$ & $27 \cdot 7$ & $4 \cdot 0$ & & $27 \cdot 5$ & $4 \cdot 0$ & & $27 \cdot 6$ & 4.4 & & $27 \cdot 5$ & 3.8 & \\
\hline Number of anti-hypertensive medications & 1.7 & 0.9 & & $1 \cdot 7$ & 0.9 & & $1 \cdot 7$ & 0.9 & & $1 \cdot 7$ & 0.9 & \\
\hline
\end{tabular}


Table 2. Effect of supplementation with $n-3$ fatty acids or B-vitamins on blood pressure (BP) measurements

\begin{tabular}{|c|c|c|c|c|c|c|c|c|c|c|c|c|c|}
\hline \multirow[b]{2}{*}{ Supplementation group } & \multirow[b]{2}{*}{ Model } & \multicolumn{3}{|c|}{ Systolic BP } & \multicolumn{3}{|c|}{ Diastolic BP } & \multicolumn{3}{|c|}{ Mean BP } & \multicolumn{3}{|c|}{ Pulse BP } \\
\hline & & $\beta$ & SD & $P$ & $\beta$ & SD & $P$ & $\beta$ & SD & $P$ & $\beta$ & SD & $P$ \\
\hline \multirow[t]{2}{*}{$n-3$ Fatty acids } & Crude & 0.04 & 0.28 & NS & -0.21 & 0.18 & NS & -0.13 & 0.20 & NS & 0.24 & 0.20 & NS \\
\hline & Adjusted & -0.06 & 0.44 & NS & 0.06 & 0.29 & NS & 0.007 & 0.31 & NS & -0.08 & 0.33 & NS \\
\hline \multirow[t]{2}{*}{ B-vitamins } & Crude & 0.44 & 0.29 & NS & 0.20 & 0.18 & NS & 0.28 & 0.20 & NS & 0.24 & 0.20 & NS \\
\hline & Adjusted & 0.14 & 0.45 & NS & 0.40 & 0.30 & NS & 0.29 & 0.32 & NS & -0.18 & 0.34 & NS \\
\hline \multirow[t]{2}{*}{ B-vitamins $+n-3$ fatty acids } & Crude & -0.03 & 0.29 & NS & -0.06 & 0.18 & NS & -0.05 & 0.20 & NS & 0.02 & 0.20 & NS \\
\hline & Adjusted & -0.68 & 0.45 & NS & -0.12 & 0.30 & NS & -0.32 & 0.32 & NS & -0.51 & 0.33 & NS \\
\hline
\end{tabular}

associated with a significant increase in median plasma EPA (by 79\%) and in median DHA (by 18\%) compared with the placebo at 1 year of intervention ${ }^{(20)}$.

No intervention effect was observed with either B-vitamins, $n-3$ fatty acids or their combination on change in systolic $\mathrm{BP}$, diastolic BP, mean $\mathrm{BP}$ or pulse $\mathrm{BP}$ in crude and adjusted models (Table 2). BP change was not associated with change in homocysteine concentration in any of the groups in all the regression models examined $(P>0 \cdot 10$; data not shown).

Fig. 1 illustrates the evolution of BP over the study period for combined $n-3$ fatty acids groups $v$. placebo (for $n-3$ fatty acids; Fig. 1(a)) and for the combined B-vitamins groups $v$. placebo (for B-vitamins; Fig. 1(b)). There was no significant effect of supplementation with either $n-3$ fatty acids or B-vitamins on systolic BP, diastolic BP, mean BP and pulse BP.

\section{Discussion}

In this secondary prevention trial with patients with established CVD, no effect on BP of supplementation for 5 years with either $n$-3 PUFA or B-vitamins was found. This result is consistent with the main analysis of the trial not showing any effect of the supplementation on the recurrence of cardiovascular or neurovascular events ${ }^{(20)}$. This finding was unchanged when analyses were restricted to individuals with hypertension at baseline.

Supplementation with B-vitamins significantly reduced homocysteine concentrations. The change in BP between successive examinations was not associated with change in homocysteine concentration in any of the supplementation groups.

Several studies have investigated the relationship between $n-3$ fatty acid supplementation and BP and found heterogeneous results ${ }^{(5,6,10,11,21,22)}$. In a meta-analysis of thirty-six trials, Geleijnse et $a l .{ }^{(5)}$ reported a reduction in both systolic and diastolic BP due to supplementation in $n$ - 3 fatty acids. Previous meta-analyses ${ }^{(10,21)}$ also found that supplementation with fish oil slightly lowered BP. However, most studies in these meta-analyses used relatively high doses of $n-3$ fatty acids and were short in duration (most lasted for $<3$ months). A more recent review from the Cochrane Collaboration $^{(6)}$ based on the results from seven studies (2743 participants in total), with a duration of at least 6 months, did not show any effect of $n-3$ supplementation on either systolic or diastolic BP. This non-significant result was confirmed after
4 years of supplementation ( $1 \mathrm{~g} / \mathrm{d}$ of $n-3$ fatty acids) in the Gruppo Italiano per lo Studio della Sopravvivenza nell'Infarto Miocardico-Heart Failure trial (patients with previous heart failure, $n$ 6975) $)^{(22)}$.

We did not find any BP-lowering effect attributable to $n-3$ fatty acids at dietary doses in the SU.FOL.OM3 study. Apart from a true absence of effect, several hypotheses could explain this finding. First, the doses used in the present study were dietary doses and thus relatively small. However, the plasma concentrations of EPA and DHA obtained after supplementation with $n$-3 fatty acids after 1 year of intervention were similar to those observed customarily in high fish consumers in France, i.e. those in the highest quartile of fish consumption in a national sample in the SU.VI.MAX study conducted in our laboratory (S Hereberg and P Galan, unpublished results), where fatty acids were determined in the same manner as in the present study. We could not compare these concentrations with those described in the literature due to differences in the populations examined $^{(23,24)}$, type and dose of supplementation ${ }^{(25)}$, as well as variability in the analytical methods for fatty acids. It may be speculated that even higher circulating levels need to be achieved in order to observe protective effects from a secondary prevention perspective. Second, the description of a positive effect in short-term trials ${ }^{(5,10,21)}$ could also indicate that the BP-lowering effect of $n-3$ fatty acids is more pronounced in the short term (less than a few months). Due to the lack of literature on supplementation with $n$ - 3 fatty acids for $>4$ years, whether a longer-term effect with higher doses of $n-3$ fatty acids could be seen cannot be excluded. Finally, the vasodilative properties of $n$ - 3 fatty acids could be inefficient on arteries already degraded by atherosclerosis, explaining their lack of an anti-hypertensive effect in secondary prevention.

With regard to the potential effect of B-vitamins, and particularly folate, several studies and meta-analyses have reported a positive effect on BP reduction in both primary and secondary prevention trials lasting between a few weeks and a few years ${ }^{(7,9,13,15)}$. However, we did not observe any relationship between B-vitamins supplementation at dietary doses, which are much smaller than those used in previous studies, and a change in BP. The present findings suggest that dietary doses of $\mathrm{B}$-vitamins are insufficient to induce a reduction in $\mathrm{BP}$; the dose-effect relationship needs further examination in future studies.

We also studied whether a change in the homocysteine level, possibly induced by folate, was associated with BP 

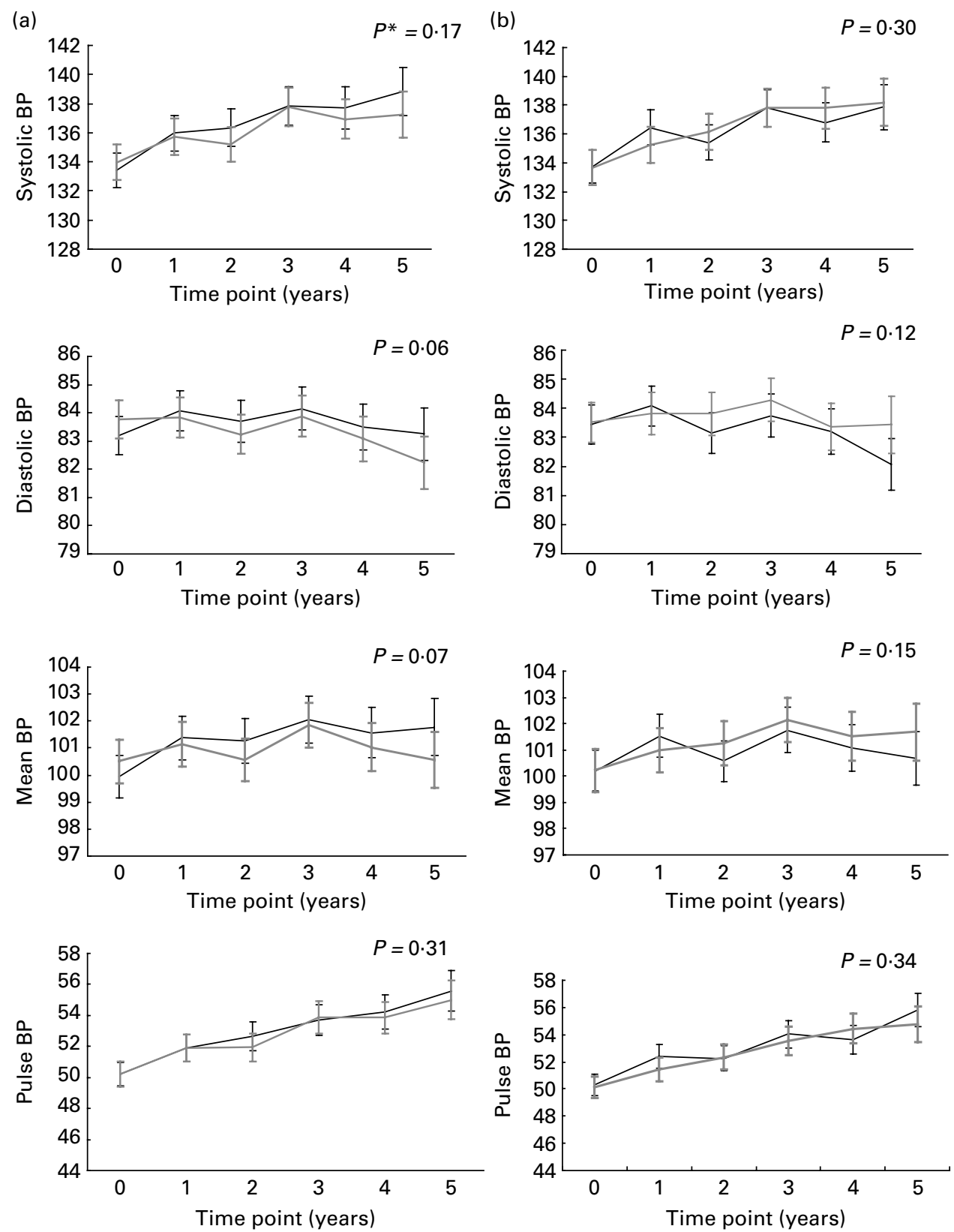

Fig. 1. Evolution of systolic, diastolic, mean and pulse blood pressure (BP) in the $n-3(-)$ - and (b) B-vitamins ( -$)$-supplemented groups and the corresponding placebos $(-)$ over the 5 years follow-up. Values are means with $95 \% \mathrm{Cl}$ represented by vertical bars. ${ }^{*} P$ for trend obtained with $t$ statistic from the mixed linear models.

change. Much evidence suggests that supplementation with folic acid or folate can lower homocysteine levels ${ }^{(19)}$. Furthermore, a positive association between plasma homocysteine levels and BP or hypertension has been described in some cross-sectional studies ${ }^{(9,17,18,26-29)}$; even though others have reported opposite results ${ }^{(30-32)}$. Certain primary and secondary intervention trials, varying from a few weeks to 2 years, have also described the BP-lowering effect with folic acid supplementation, which was associated with a decrease in homocysteine levels ${ }^{(7,9,13-16)}$; this effect seems to occur only with high doses of folate or folic acid ( $>5 \mathrm{mg} / \mathrm{d}$ ). In contrast, consistent with the present findings, others have reported no effect of supplementation with B-vitamins (including folic acid/folate) on BP. In an intervention trial lasting 2 years involving 276 healthy older adults with hyperhomocysteinaemia, who took either a daily supplement of B-vitamins (folate $(1 \mathrm{mg})$, vitamin $\mathrm{B}_{12}(500 \mu \mathrm{g})$ and vitamin $\mathrm{B}_{6}(10 \mathrm{mg})$ ) or a placebo ${ }^{(33)}$, no effect on BP was observed. Similarly, in the large Vitamin Intervention for Stroke Prevention (VISP) trial (including 3361 individuals with a prior history of a stroke) using B-vitamins at either a low dose (200 $\mu$ g pyridoxine, $6 \mu \mathrm{g}$ cobalamin and $20 \mu \mathrm{g}$ folic acid) or a high dose (25 mg pyridoxine, $0.4 \mathrm{mg}$ cobalamin and $2.5 \mathrm{mg}$ folic acid), BP did not change following 2 years of supplementation ${ }^{(8)}$. Taken together, the mixed findings in the literature suggest that the lack of a beneficial effect on BP observed in the 
present study is most probably related to the low dose used. The lack of association between change in homocysteine and change in BP in the present study could be related to arterial stiffness in the study sample involving patients with established CVD. It has been shown that in hypertensive patients, the levels of homocysteine are strongly and independently correlated to arterial stiffness ${ }^{(34,35)}$. Plasma homocysteine and aortic pulse wave velocity are higher in patients presenting with clinical vascular disease. It is possible that the effect of a change in homocysteine concentrations on reduction in $\mathrm{BP}$ is modified by the degree of arterial stiffness and this needs further examination.

Certain limitations of the study must be considered. The study involved low doses of supplements; thus it cannot answer whether an effect of B-vitamins or $n-3$ fatty acids would be observed at higher doses in long-term trials. The study was conducted in a double-blind manner, which should have avoided a systematic bias induced by change in diet and/or supplementation practices of the participants, but this cannot be ruled out. In order to address this, we examined the evolution of the plasma concentrations of B-vitamins and $n-3$ fatty acids in the study. The groups did not differ on either B-vitamins or $n-3$ fatty acids at baseline, and significant increases were observed in B-vitamins and $n-3$ fatty acids in the groups receiving the corresponding supplements and not in any other groups ${ }^{(20)}$. Thus dietary changes, if any, did not confound the intervention design.

The present study has several strengths. First, to our knowledge, the present intervention trial is one of the few that had a long duration (median 4.7 years). Second, its aim was to test the benefit of low doses of supplements that could be achieved via dietary means. The study design involving a double-blind, randomised controlled trial is another strength. Thus, the findings of a lack of benefit of low-dose supplementation with B-vitamins or $n-3$ fatty acids in a population with established CVD are novel and deserve confirmation in other populations.

In summary, the present results do not support any effect of supplementation with B-vitamins or $n$ - 3 fatty acids at dietary doses on BP in individuals with a prior history of CVD.

\section{Acknowledgements}

We thank the health professionals, staff at the coordinating centre and study centres as well as the patients who participated in the SU.FOL.OM3 trial. We also acknowledge Gwenael Monot for data management. The SU.FOL.OM3 study was funded by the French Ministry of Research (grant R02010JJ), the Ministry of Health (Direction Générale de la Santé; DGS), Sodexo, Candia, Unilever, Danone, Roche Laboratories, Merck EPROVA GS and Pierre Fabre Laboratories. None of the funding organisations had any involvement in the design and conduct of the study, or in the collection, management, analysis and interpretation of the data, or in the preparation, review and approval of the manuscript. None of the authors has any competing interests, and all are independent of the funding bodies. P. G. and S. H. designed the study; P. G., S. H. and J. B. conducted the study; F. S. d. E. analysed the data and wrote the manuscript with N. A.; A.-C. V., C. J. and N. A. assisted with the data analysis; F. S. d. E. had primary responsibility for the final content. All authors contributed to the manuscript development, and read and approved the final manuscript.

\section{References}

1. He FJ \& MacGregor GA (2007) Salt, blood pressure and cardiovascular disease. Curr Opin Cardiol 22, 298-305.

2. MacMahon S, Peto R, Cutler J, et al. (1990) Blood pressure, stroke, and coronary heart disease. Part 1, prolonged differences in blood pressure: prospective observational studies corrected for the regression dilution bias. Lancet 335, $765-774$.

3. Forman JP, Rimm EB, Stampfer MJ, et al. (2005) Folate intake and the risk of incident hypertension among US women. JAMA 293, 320-329.

4. Knapp HR (1991) Hypotensive effects of omega 3 fatty acids: mechanistic aspects. World Rev Nutr Diet 66, 313-328.

5. Geleijnse JM, Giltay EJ, Grobbee DE, et al. (2002) Blood pressure response to fish oil supplementation: metaregression analysis of randomized trials. $J$ Hypertens $\mathbf{2 0}$, 1493-1499.

6. Hooper L, Thompson RL, Harrison RA, et al. (2004) Omega 3 fatty acids for prevention and treatment of cardiovascular disease. The Cochrane Database of Systematic Reviews 2004 issue 4, CD003177.

7. McRae MP (2009) High-dose folic acid supplementation effects on endothelial function and blood pressure in hypertensive patients: a meta-analysis of randomized controlled clinical trials. J Chiropr Med 8, 15-24.

8. Toole JF, Malinow MR, Chambless LE, et al. (2004) Lowering homocysteine in patients with ischemic stroke to prevent recurrent stroke, myocardial infarction, and death: the Vitamin Intervention for Stroke Prevention (VISP) randomized controlled trial. JAMA 291, 565-575.

9. van Dijk RA, Rauwerda JA, Steyn M, et al. (2001) Long-term homocysteine-lowering treatment with folic acid plus pyridoxine is associated with decreased blood pressure but not with improved brachial artery endothelium-dependent vasodilation or carotid artery stiffness: a 2-year, randomized, placebo-controlled trial. Arterioscler Thromb Vasc Biol 21, 2072-2079.

10. Appel LJ, Miller ER III, Seidler AJ, et al. (1993) Does supplementation of diet with 'fish oil' reduce blood pressure? A meta-analysis of controlled clinical trials. Arch Intern Med 153, 1429-1438.

11. Cicero AF, Ertek S \& Borghi C (2009) Omega-3 polyunsaturated fatty acids: their potential role in blood pressure prevention and management. Curr Vasc Pharmacol 7, $330-337$.

12. Mori TA \& Woodman RJ (2006) The independent effects of eicosapentaenoic acid and docosahexaenoic acid on cardiovascular risk factors in humans. Curr Opin Clin Nutr Metab Care 9, 95-104.

13. Cagnacci A, Cannoletta M \& Volpe A (2009) High-dose short-term folate administration modifies ambulatory blood pressure in postmenopausal women. A placebo-controlled study. Eur J Clin Nutr 63, 1266-1268.

14. Mangoni AA, Sherwood RA, Swift CG, et al. (2002) Folic acid enhances endothelial function and reduces blood pressure in smokers: a randomized controlled trial. J Intern Med 252, 497-503. 
15. Papandreou D, Malindretos P, Arvanitidou M, et al. (2010) Homocysteine lowering with folic acid supplements in children: effects on blood pressure. Int J Food Sci Nutr 61, $11-17$.

16. Williams C, Kingwell BA, Burke K, et al. (2005) Folic acid supplementation for $3 \mathrm{wk}$ reduces pulse pressure and large artery stiffness independent of MTHFR genotype. Am J Clin Nutr 82, 26-31.

17. Nygard O, Vollset SE, Refsum H, et al. (1995) Total plasma homocysteine and cardiovascular risk profile. The Hordaland Homocysteine Study. JAMA 274, 1526-1533.

18. Kahleova R, Palyzova D, Zvara K, et al. (2002) Essential hypertension in adolescents: association with insulin resistance and with metabolism of homocysteine and vitamins. Am J Hypertens 15, 857-864.

19. Homocysteine Lowering Trialists' Collaboration (2005) Dosedependent effects of folic acid on blood concentrations of homocysteine: a meta-analysis of the randomized trials. Am J Clin Nutr 82, 806-812.

20. Galan P, Kesse-Guyot E, Czernichow S, et al. (2010) B-vitamins, $n-3$ polyunsaturated fatty acids and risk of cardiovascular events: the SU.FOL.OM3 trial. BMJ 341, c6273.

21. Morris MC, Sacks F \& Rosner B (1993) Does fish oil lower blood pressure? A meta-analysis of controlled trials. Circulation 88, 523-533.

22. Tavazzi L, Maggioni AP, Marchioli R, et al. (2008) Effect of $n-3$ polyunsaturated fatty acids in patients with chronic heart failure (the GISSI-HF trial): a randomised, doubleblind, placebo-controlled trial. Lancet 372, 1223-1230.

23. Ayer JG, Harmer JA, Xuan W, et al. (2009) Dietary supplementation with $n-3$ polyunsaturated fatty acids in early childhood: effects on blood pressure and arterial structure and function at age 8 y. Am J Clin Nutr 90, 438-446.

24. Mayneris-Perxachs J, Bondia-Pons I, Serra-Majem L, et al. (2010) Long-chain $n-3$ fatty acids and classical cardiovascular disease risk factors among the Catalan population. Food Chem 119, 54-61.

25. Ishikawa Y, Yokoyama M, Saito Y, et al. (2010) Preventive effects of eicosapentaenoic acid on coronary artery disease in patients with peripheral artery disease. Circ $J \mathbf{7 4 ,}$ 1451-1457.

26. Budge MM, de Jager C, Hogervorst E, et al. (2002) Total plasma homocysteine, age, systolic blood pressure, and cognitive performance in older people. $\mathrm{J} \mathrm{Am}$ Geriatr Soc 50, 2014-2018.

27. Lim U \& Cassano PA (2002) Homocysteine and blood pressure in the Third National Health and Nutrition Examination Survey, 1988-1994. Am J Epidemiol 156, 1105-1113.

28. Refsum H, Nurk E, Smith AD, et al. (2006) The Hordaland Homocysteine Study: a community-based study of homocysteine, its determinants, and associations with disease. $J$ Nutr 136, 1731-1740.

29. Sutton-Tyrrell K, Bostom A, Selhub J, et al. (1997) High homocysteine levels are independently related to isolated systolic hypertension in older adults. Circulation 96, 1745-1749.

30. Dinavahi R, Cossrow N, Kushner H, et al. (2003) Plasma homocysteine concentration and blood pressure in young adult African Americans. Am J Hypertens 16, 767-770.

31. Fakhrzadeh H, Ghotbi S, Pourebrahim R, et al. (2005) Plasma homocysteine concentration and blood pressure in healthy Iranian adults: the Tehran Homocysteine Survey (20032004). J Hum Hypertens 19, 869-876.

32. Sundstrom J, Sullivan L, D'Agostino RB, et al. (2003) Plasma homocysteine, hypertension incidence, and blood pressure tracking: the Framingham Heart Study. Hypertension $\mathbf{4 2}$ 1100-1105.

33. McMahon JA, Skeaff CM, Williams SM, et al. (2007) Lowering homocysteine with $\mathrm{B}$ vitamins has no effect on blood pressure in older adults. J Nutr 137, 1183-1187.

34. Blacher J, Demuth K, Guerin AP, et al. (1998) Influence of biochemical alterations on arterial stiffness in patients with end-stage renal disease. Arterioscler Thromb Vasc Biol 18, 535-541.

35. Bortolotto LA, Safar ME, Billaud E, et al. (1999) Plasma homocysteine, aortic stiffness, and renal function in hypertensive patients. Hypertension 34, 837-842. 